Ophthalmologica

\title{
Spontaneous Reattachment of the Margins of a Macular Retinal Pigment Epithelium Tear: Optical Coherence Tomography Documentation of a Case
}

\author{
Alfredo Pece ${ }^{a} \quad$ Lucia Vitale $^{a}$ Paolo Milanib ${ }^{b}$ Luisa Pierro $^{c}$ \\ ${ }^{a}$ Ospedale Vizzolo-Predabissi, Melegnano, e ${ }^{b}$ Ospedale Fatebenefratelli-Oftalmico e ${ }^{c}$ Ospedale San Raffaele, \\ Università Vita e Salute, Milano, Italia
}

\section{Key Words}

Age-related macular degeneration - Retinal pigment epithelium tear, reattachment $\cdot$ Optical coherence tomography

\begin{abstract}
Purpose: To document by optical coherence tomography (OCT) the reattachment of the margins of a retinal pigment epithelium (RPE) tear. Methods: Single case report, documented by OCT scans, autofluorescence and fluorescein angiography. Results: A 67-year-old male presented with a spontaneous RPE tear due to age-related macular degeneration in his right eye. Three months later we observed that the focal RPE tear had healed and there was a new intraretinal fluid, well documented by OCT imaging. Conclusions: OCT scans show the reattachment of the margins of an RPE tear healed by tissue remodelling, and illustrate how the disease can recur.

Copyright $\odot 2009$ S. Karger AG, Basel
\end{abstract}

\section{Introduction}

Tear of the retinal pigment epithelium (RPE) was first described as possible complication of a vascularized RPE detachment associated with age-related macular degen- eration (AMD) [1-3]. A tear may arise spontaneously or in relation to various treatments, such as laser photocoagulation, photodynamic therapy or intravitreal injection of anti-vascular-endothelial-growth-factor agents [4-7].

The pattern and course of RPE tears have been studied in the past by fluorescein fundus angiography, autofluorescence (AF), fundus ophthalmoscopy, stereo colour photography and more recently by optical coherence tomography (OCT) [8-13]. Hoskin et al. [1] described the fluorescein findings of reattachment of the free margin to Bruch's membrane, a few days or weeks after the tear.

Vitreomacular traction in the foveal area has been postulated to contribute to the subsequent formation of RPE tears via mechanical or cell-mediated pathways [13].

We describe the OCT documentation of a spontaneous AMD-associated macular RPE tear, with subsequent reattachment of the margins.

\section{Case Report}

In July 2007, a 67-year-old man came to our observation complaining of decreased vision and metamorphopsia in his right eye, starting 1 month earlier. Visual acuity was 20/320. The left eye had some drusen at the posterior pole. Right eye fundus biomicroscopy disclosed a well-demarcated area of RPE loss involving

\section{KARGER}

Fax +41613061234 E-Mail karger@karger.ch www.karger.com (c) 2009 S. Karger AG, Basel

$0030-3755 / 10 / 2243-0159 \$ 26.00 / 0$

Accessible online at:

www.karger.com/oph
Alfredo Pece

Via N. Bixio 3

IT-20129 Milano (Italy)

Tel. +39 022951 1378, Fax +39 0220422262

E-Mail Pece.retina@mclink.it 

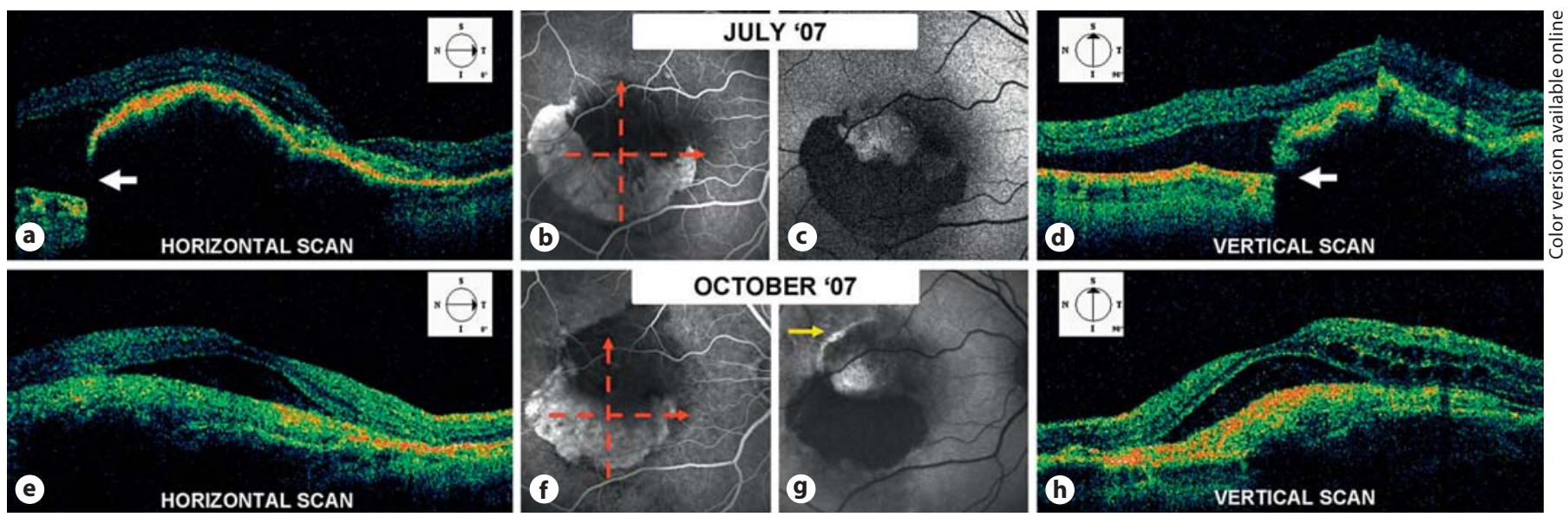

Fig. 1. Dashed lines (red in the online version) indicate OCT scan correspondence in the macula. Circled arrows illustrate the orientation of the scan. a-d Images taken in July 2007. OCT horizontal and vertical tomograms (a, d) show a large RPE detachment with focal disruption in the RPE layer (white arrows), which corresponds to the clinical finding of an RPE tear, and subretinal fluid. b Fluorescein angiography discloses a hyperfluorescent area with no leakage (window defect) in the lower part of the macular lesion. The upper part is hypofluorescent because of the blocking effect of the retracted RPE. c AF evidences a large black area in the lower part of the lesion corresponding to the absence

the fovea with evidence of the underlying choroid. The superior part of the macula appeared elevated with signs of RPE detachment. Fluorescein angiography (HRA2, Heidelberg Engineering, Germany) indicated the absence of RPE in the inferior part of the lesion (fig. 1b), corresponding to a clear-cut area of fluorescence with no leakage (window defect). There was hypofluorescence in the upper part of the lesion due to the blocking effect of the retracted RPE and the RPE detachment. AF showed up a large black area in the inferior part of the lesion, corresponding to the lack of the RPE layer and a diffuse hyper-AF in the superior part of the lesion (fig. 1c).

OCT horizontal and vertical scans (OCT 3, Stratus, Carl Zeiss Meditech, Dublin, Calif., USA) confirmed the RPE detachment, focal disruption in the RPE layer corresponding to the tear, and subretinal fluid adjacent to the tear (fig. 1a, d).

Observation was suggested, and 3 months later the patient returned for scheduled control. Visual acuity in the right eye was 20/400 and fundus biomicroscopy unchanged.

Fluorescein angiography indicated enlargement of the superior RPE detachment area (fig. 1f), and AF disclosed a halo of a lightly increased shadow effect surrounding the lesion nasally and superiorly. The fovea area acquired hyper-AF, and, similarly, a hyperautofluorescent band along the upper edge of the lesion became evident (fig. 1g).

OCT horizontal and vertical foveal scans showed the complete reattachment of the ripped RPE margins, and no focal disruption was visible anymore. Moreover, a long hyperreflective RPE band, an amount of fluid and some cystoid changes in the neurosensory retina were evident (fig. le, h).

of an RPE layer. e-h Images taken in October 2007. OCT horizontal and vertical scans $(\mathbf{e}, \mathbf{h})$ indicate the complete reattachment of the ripped margins of the RPE. Some intraretinal fluid and cystoid macular oedema are evident. $f$ Fluorescein angiography indicated enlargement of the superior RPE detachment area. g AF shows a hyperautofluorescent band (white arrow; yellow in the online version) along the upper edge of the lesion and increased $\mathrm{AF}$ in correspondence with the foveal region. A light hypo-AF halo surrounds the lesion nasally and superiorly, due, very probably, to the presence of subretinal fluid.

\section{Discussion}

RPE tear occurs spontaneously in about $10 \%$ of vascularized AMD-related pigment epithelial detachments and may also be related to various treatments, such as laser photocoagulation, photodynamic therapy or intravitreal injections [5-7]. The tear occurs at the edge of the pigment epithelial detachment, most often along the temporal margin, and the detached RPE usually retracts into fine folds parallel to the edge of the tear, leaving an area of bare Bruch's membrane and remaining free in the subepithelial space for a few days $[1,2,4]$. Spontaneous flattening of pigment epithelial detachment is frequent after RPE tear; reattachment of the free RPE margin to Bruch's membrane, often at a new site, has been described [1].

An overexpression of 'new tissue' can occur at the tear site, closing the tear and restoring the continuity of the RPE layer. Repopulation of the RPE after retinal pigment tear documented with OCT has recently been described [12]. Similarly, in our case OCT clearly documented RPE margin reattachment, corresponding to a hyperreflective RPE band (fig. 1h). AF imaging in the ripped area still showed no AF (fig. 1g), due to the lack of RPE. In our opinion this could be explained as a different biochemi- 
cal composition of repopulated RPE. We do know, in fact, that $\mathrm{AF}$ is mainly determined by lipofuscin contained in RPE cells, that probably could be missing in the renewed $\mathrm{RPE}$, at least in the first remodelling phase. In fact as Gass [2] described after some weeks the bare Bruch's membrane becomes covered by fibrous metaplastic tissue derived from RPE cell proliferation from the edge of the defect not involved in the original detachment. In other cases, especially when the defect is small, the bare Bruch's membrane becomes covered by a layer of relatively normal-looking, hypopigmented pigment epithelium derived from cells sliding from the margin of the defect $[1$, 4]; in a few instances, the appearance does not change after the initial tear, in which case Bruch's membrane presumably either remains nude or is covered by non-pigmented cells $[1,2,4]$.

Peiretti et al. [12] described some peculiar AF findings at the site of the tear and assumed that hyper-AF may indicate a curled or redundant RPE layer. In our case there was a band of hyper-AF along the upper edge of the lesion (fig. 1, yellow arrow) and an oval hyper-AF area in correspondence with the foveal area (fig. $1 \mathrm{~g}$ ). These changes seemed related, in our opinion, to a reaction of the RPE lipofuscin remodelling in some parts of the lesion.

Also we noted that a recurrent exudation, proved by intraretinal fluid and very probably light hypo-AF, can occur.
Certainly, imaging accuracy and alignment strategies are fundamental when studying such lesions. RPE reattachment concordance was firstly observed and then confirmed by one of the authors, L.P., who skilfully performed several scans of all the complete macular area. Confrontation point by point with the 3 -month earlier examination was possible due to good patient collaboration. Unfortunately we did not dispose of spectral-domain OCT that would have contributed to add more definition and particulars in studying the lesion. Similarly, the correspondence of the OCT scans with the single portion of the macula investigated would have been more precise with high-speed scanning.

However, in spite of the lower reproducibility and limitation of time domain, compared to the new spectral-domain technology (not available at the time of patient examination), we think that in this case the Stratus OCT 3 correctly documented RPE margin reattachment after a tear in AMD. The findings enabled us to understand the mechanism leading to the repair processes and illustrated the possibility of reactivation of the disease, even after the tear had healed.

\section{Acknowledgement}

This work was supported by the Fondazione Retina 3000, MiThis

\section{References}

$\checkmark 1$ Hoskin A, Bird AC, Sehmi K: Tears of de tached retinal pigment epithelium. $\mathrm{Br} \mathrm{J} \mathrm{Oph-}$ thalmol 1981;65:417-422.

2 Gass JD: Pathogenesis of tears of the retinal pigment epithelium. Br J Ophthalmol 1984; 68:513-519.

3 Chuang EL, Bird AC: Repair after tear of the retinal pigment epithelium. Eye 1988;2:106113.

4 Chang LK, Sarraf D: Tears of the retinal pigment epithelium. an old problem in a new era. Retina 2007;27:523-534.

$\checkmark 5$ Pece A, Introini U, Bottoni F, Brancato R: Acute retinal pigment epithelial tear after photodynamic therapy. Retina 2001;21:661665.

\footnotetext{
-6 Chan CK, Meyer CH, Gross JG, Abraham P, Nuthi AS, Kokame GT, Lin SG, Rauser ME, Kaiser PK: Retinal pigment epithelial tears after intravitreal bevacizumab injection for neovascular age-related macular degeneration. Retina 2007;27:541-551.

7 Chan CK, Lin SG: Retinal pigment epithelial tear after ranibizumab therapy for subfoveal fibrovascular pigment epithelial detachment. Eur J Ophthalmol 2007;17:674-676.

-8 Giovannini A, Amato G, Mariotti C, Scassellati-Sforzolini B: Optical coherence tomography in the assessment of retinal pigment epithelial tear. Retina 2000;20:37-40.

9 Krishan NR, Chandra SR, Stevens TS: Diagnosis and pathogenesis of retinal pigment epithelial tears. Am J Ophthalmol 1985;15: 698-707.
}

10 Cantrill HL, Ramsay RC, Knobloch WH: Rips in the pigment epithelium. Arch Ophthalmol 1983;101:1074-1079.

11 Karadimas P, Paleokastritis GP, Bouzas EA: Fundus autofluorescence imaging findings in retinal pigment epithelial tear. Eur J Ophthalmol 2006;16:767-769.

12 Peiretti E, Iranmanesh R, Lee JJ, Klancnik JM Jr, Sorenson JA, Yannuzzi LA: Repopulation of the retinal pigment epithelium after pigment epithelial rip. Retina 2006;26:10971099.

13 Meyer CH, Toth CA: Retinal pigment epithelial tear with vitreomacular attachment: a novel pathogenic feature. Graefes Arch Clin Exp Ophthalmol 2001;239:325-333. 